University of Wollongong

Research Online

Faculty of Engineering - Papers (Archive)

Faculty of Engineering and Information

Sciences

2012

\title{
Characterization of a novel diamond-based microdosimeter prototype for radioprotection applications in space environments
}

Jeremy A. Davis

University of Wollongong, jad028@uowmail.edu.au

Kumaravelu Ganesan

University Of Melbourne

Andrew D. C Alves

University Of Melbourne

Susanna Guatelli

University of Wollongong, susanna@uow.edu.au

Marco Petasecca

University of Wollongong, marcop@uow.edu.au

See next page for additional authors

Follow this and additional works at: https://ro.uow.edu.au/engpapers

Part of the Engineering Commons

https://ro.uow.edu.au/engpapers/5267

\section{Recommended Citation}

Davis, Jeremy A.; Ganesan, Kumaravelu; Alves, Andrew D. C; Guatelli, Susanna; Petasecca, Marco; Livingstone, Jayde; Lerch, Michael L. F; Prokopovich, Dale A.; Reinhard, Mark I.; Siegele, Rainer M.; Prawer, Steven; Jamieson, David; Kuncic, Zdenka; Pisacane, Vincent L.; Dicello, John F.; Ziegler, James; Zaider, Marco; and Rosenfeld, Anatoly B.: Characterization of a novel diamond-based microdosimeter prototype for radioprotection applications in space environments 2012, 3110-3116.

https://ro.uow.edu.au/engpapers/5267

Research Online is the open access institutional repository for the University of Wollongong. For further information contact the UOW Library: research-pubs@uow.edu.au 


\section{Authors}

Jeremy A. Davis, Kumaravelu Ganesan, Andrew D. C Alves, Susanna Guatelli, Marco Petasecca, Jayde Livingstone, Michael L. F Lerch, Dale A. Prokopovich, Mark I. Reinhard, Rainer M. Siegele, Steven Prawer, David Jamieson, Zdenka Kuncic, Vincent L. Pisacane, John F. Dicello, James Ziegler, Marco Zaider, and Anatoly B. Rosenfeld 


\title{
Characterisation of a Novel Diamond-Based Microdosimeter Prototype for Radioprotection Applications in Space Environments
}

\begin{abstract}
Jeremy Davis Student Member IEEE, Kumaravelu Ganesan, Andrew Alves, Susanna Guatelli Member IEEE, Marco Petasecca, Jayde Livingstone Student Member IEEE, Michael L.F. Lerch Member IEEE, Dale A. Prokopovich Member IEEE, Mark I. Reinhard Member IEEE, Rainer Siegele, Steven Prawer, David Jamieson, Zdenka Kuncic, Vincent L. Pisacane, John F. Dicello, James Ziegler, Marco Zaider and Anatoly B. Rosenfeld Senior Member IEEE
\end{abstract}

\begin{abstract}
This paper is dedicated to the characterization of a novel diamond microdosimeter prototype with $3 D$ sensitive volumes produced by high energy boron implantation. Diamond has been chosen in order to further improve solid state based microdosimeter in terms of radiation hardness and tissue equivalency. IBIC measurements were undertaken to determine the charge collection efficiency map of the device. It was demonstrated that the proposed ion implantation technology allows for the formation of an array of well defined 3D SVs. A Geant4 application was developed to explain the effect of $\mathrm{Al}$ electrode thickness on observed anomaly in deposited energy. Specifics of the results and an update on the current status of the project is presented.
\end{abstract}

Index Terms - Radiation protection, space radiation environment, diamond, microdosimetry.

\section{INTRODUCTION}

$\mathrm{M}$ ICRODOSIMETRY is a methodology that involves the measurement or calculation of stochastic energy deposition distributions in a micron size sensitive volume (SV) within any arbitrary mixed radiation field. This culminates in the production of a lineal energy spectrum which when compared with a well established quality coefficient can be used to determine dose equivalent [1]. While microdosimetry has a wide range of applications, the focus of this paper is the potential use of a diamond based

Manuscript received July 6, 2012. This work was supported by the Australian Research Council (DP1096600) and the National Space and Biomedical Research Institute, USA.

J.A. Davis, S. Guatelli, M. Petasecca, J. Livingstone, M. Lerch and A.

B.Rosenfeld are with the Centre for Medical Radiation Physics, University of Wollongong, Wollongong, NSW 2500 Australia (email: jad028@uow.edu.au, susanna@uow.edu.au,marcop@uow.edu.au, j1883@uow.edu.au and anatoly@uow.edu.au).

K. Ganesan, A.Alves, S. Prawer and D. Jamieson are with the University of Melbourne, Australia (email: kganesan@unimelb.edu.au,

s.prawer@unimelb.edu.au and d.jamieson@unimelb.edu.au).

D. A. Prokopovich, M. I. Reinhard and R. N. Siegele are with the Australian

Nuclear Science and Technology Organisation, Lucas Heights, NSW

Australia (email: dale.prokopovich@ansto.gov.au ,

mark.reinhard@ansto.gov.au and rainer.siegele@ansto.gov.au).

Z. Kuncic is with the University of Sydney, Sydney, NSW Australia (email: zdenka.kuncic@sydney.edu.au).

J. Ziegler are with the United States Naval Academy, Annapolis, MD 21402

USA (e-mail:, ziegler@SRIM.org)

M. Zaider is with the Department of Medical Physics, Memorial

Sloan-Kettering Cancer Center, New York, NY 10021 USA (e-mail: zaiderm@mskcc.org).

V. L. Pisacane and J. F. Dicello are retired from USNA USA (e-mail: diceljo@verizon.net and vincepisacane@gmail.com).

Manuscript received July 13th, 2012. microdosimeter as a radiation monitoring system for astronauts in a space environment.

Detectors used in a space radiation environment by necessity have different basic requirements than those used in regular terrestrial applications. The complex and harsh radiation environment of free space necessitates that the device be radiation hard. In addition the nature of its use assigns additional limitations upon mass as well as power consumption.

Diamond has been chosen as a candidate material to potentially supersede previous silicon based microdosimeters developed at the Centre for Medical Radiation Physics (CMRP), University of Wollongong, with its collaborative partners [2]. Diamond is well characterised with its properties well documented in the literature [3]-[9]. Radiation detectors based on diamond are attractive for space applications, due to its radiation hardness, large breakdown voltage and relative independence of their operation on temperature. In addition its low dark current, high carrier mobility and low capacitance implies both fast signal collection and low noise. With consideration of the relevant properties, diamond has clear advantages as a material to be used in the building of radiation detectors for use in a harsh radiation environment.

The purpose of this work is to establish the functionality of new fabrication techniques in diamond in relation to microdosimetry. This work will use a combination of experimental and computer based techniques to evaluate this new technology.

\section{TECHNOLOGY OF DIAMOND DETECTOR}

This novel diamond microdosimeter prototype has been designed and fabricated by the University of Melbourne in collaboration with CMRP. The microdosimeter consists of an array of SVs connected in parallel and realised on a substrate of single crystal Chemical Vapour Deposited (scCVD) diamond. ScCVD has been chosen for its high stability, better charge collection efficiency and lower dark current than the standard polyCVD diamond substrates [4]. The diamond microdosimetric detector was fabricated on ultra high purity diamond from Element Six CVD diamond with size 3.5x3.5 $\mathrm{x} 0.25 \mathrm{~mm}$ [10]. The fabrication steps are shown in Fig. 1.

The concept behind this device was to create 3D vertical wall-less SV's embedded in a diamond matrix using radiation damaged pattern to separate SVs in terms of charge collection rather than spatially separated planar diode structures or mesa structures as we utilised in our Silicon-On-Insulator microdosimeters [2]. In this case, a $2 \mathrm{MeV}$ Boron was implanted through an electron beam evaporated $4 \mu \mathrm{m}$ thick aluminium mask at a fluence of $1 \times 10^{15} \mathrm{~B} / \mathrm{cm}^{2}$ in order to 
create a buried conducting electrode structure at a $1.38 \mu \mathrm{m}$ depth. After ion implantation, annealing treatment was performed at $1425^{\circ} \mathrm{C}$ for 15 minutes in vacuum.

a)

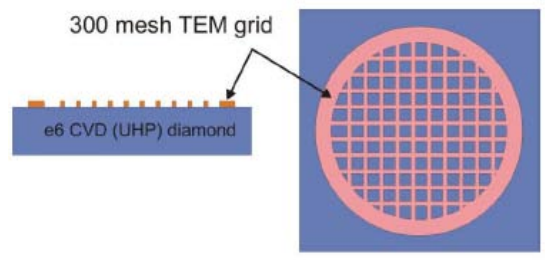

b)

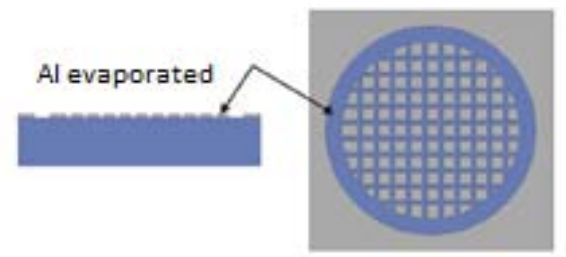

C)

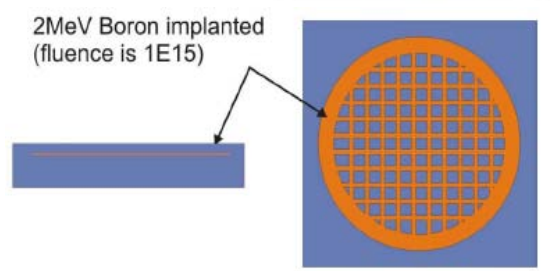

d)

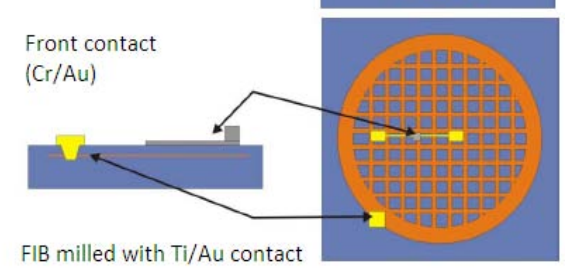

e)

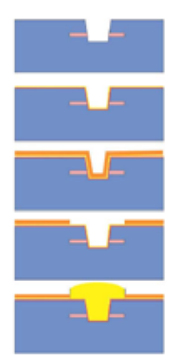

FIB milling

Ti/Gold evaporation (10/50nm)

Photoresist

Open window by photolithography

Electroforming of gold

Fig.1. Schematic of the buried boron electrode detector. a) A TEM grid with pitch $100 \mu \mathrm{m}$ is placed on a diamond substrate. b) $4 \mu \mathrm{m} \mathrm{Al}$ was evaporated through the TEM grid. c) $2 \mathrm{MeV}$ Boron was implanted at room temperature. d) Focus Ion Beam (FIB) milling followed by thermal evaporation and electroplating for making electrical connection to the buried boron electrode. e) Shows the last stage of creating the common back contact in more details

This fabrication method allows for the production of well defined sensitive volumes (SVs) as well as suitable electrical characteristics. In this design, SV regions were $150 \times 150 \times 1.38$ $\mu^{3}$. Whilst the size of the SV regions is not truly representative of true microdosimetric volumes, they do allow for testing of the fabrication concept.

The boron doped grid is connected to the gold/titanium contact that has been thermally evaporated and electroplated to be within the Focused Ion Beam (FIB) milled region seen in Fig. 1d. This represents the back contact to the buried boron implanted mesh of the device. After further annealing of the device at $800^{\circ} \mathrm{C}$ inside a vacuum, front metallisation of the device was carried out. Two types of front electrodes were evaporated onto the device. The first was multiple groups of small pads of $\mathrm{Cr} / \mathrm{Au}$ contacts connected to each other in a circular configuration. The second was a $0.5 \mu \mathrm{m}$ thick circular aluminium pad evaporated across a large region of SVs and several $\mathrm{Cr} / \mathrm{Au}$ pads which can be seen on a Fig 2 and zoomed on a Fig 3.

Fig. 2 and 3 shows an optical microscope image of the diamond microdosimeter. The circular pad is the aforementioned aluminium pad with an optical shadow to its left. The light region is a silver paste that is at the back of the diamond device and the grey is the $3.5 \times 3.5 \mathrm{~mm}^{2}$ scCVD diamond. In Fig. 3 the optical zoom is increased to depict the aluminium pad on the microdosimeter, set above the SV regions.

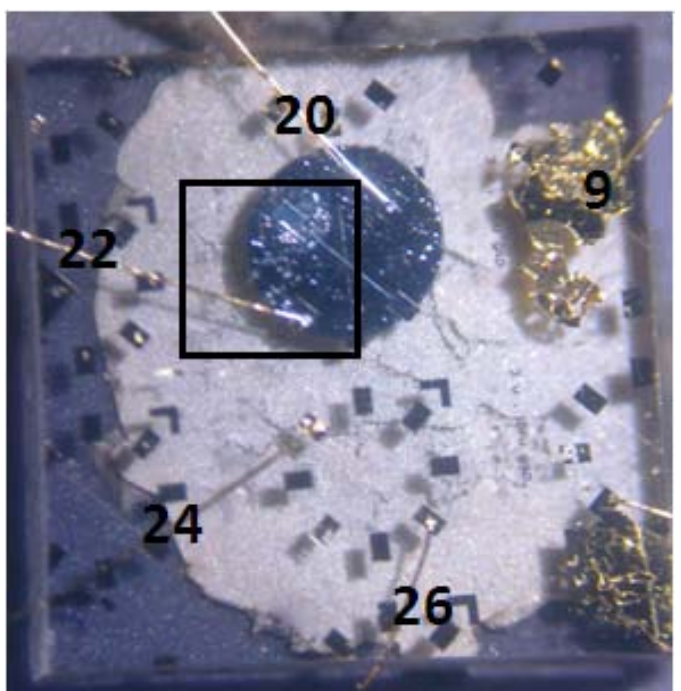

Fig. 2- Optical microscope image of the diamond $3.5 \times 3.5 \mathrm{~mm}^{2}$ microdosimeter. Two bonded wire (20 and 22) can be seen in contact with the aluminium pad and two bonded wires (24 and 26) connected to deposited $\mathrm{Cr} / \mathrm{Au}$ pads on top of the device. A square has been placed over the aluminium pad, to show the region displayed in Fig. 3. Gold contact (9) contacts to buried boron mesh as on a Fig.1e. Contacts numbers correspond to pin out of used die DIL package.

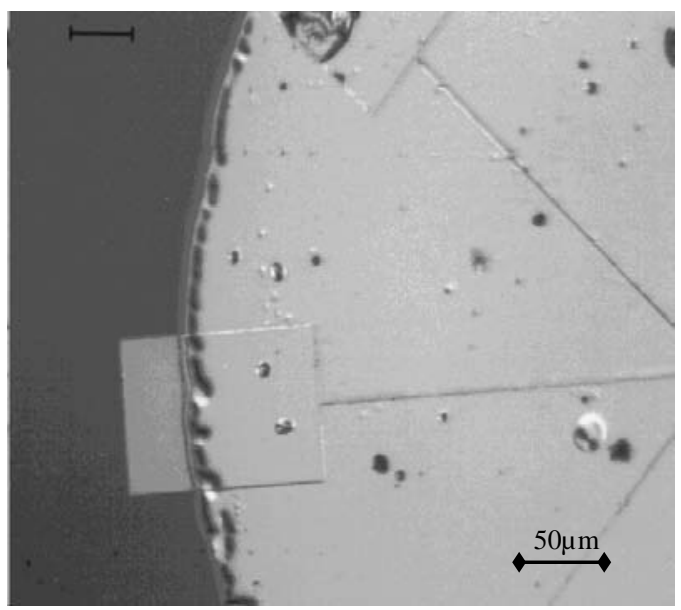

Fig.3 - Optical microscope image of the fragment of diamond microdosimeter. Magnification was increased to show the aluminium pad in greater detail.

Fig. 4 shows a cathodoluminescence image of the device taken before the Al pad evaporation and wire bonding. The region depicted in Fig. 4 can also be seen Fig. 2 in the bottom half of the image with bonded wires 24 and 26. Due to the difference in materials, the cathodoluminescence technique is 
clearly able to differentiate the different regions of importance in the device. The grey squares represent individual diamond not implanted SV's. The regions in between represent the boron implanted diamond, and finally the dark rectangles are the top side $\mathrm{Cr} / \mathrm{Au}$ contacts.

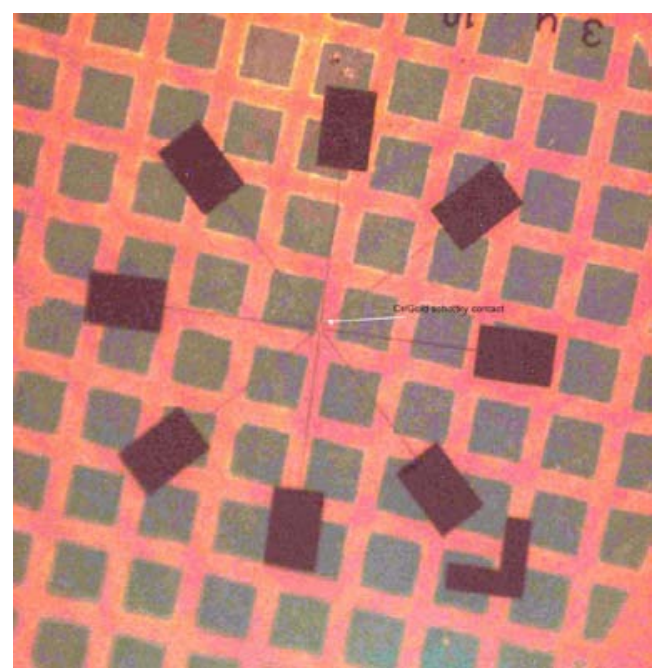

Fig. 4- Cathodoluminescence image of the diamond microdosimeter. The pure diamond SV's can clearly be seen as being separate from the boron implanted or radiation damaged regions. Cr/Au 100x200 $\mu \mathrm{m}^{2}$ pads of circular configuration are clear seen.

Material selection for front electrodes must satisfy a balance between the desirable mechanical/electronic properties and technical limitations. Front contacts will produce a change in the stopping power of ions entering the SV thus affecting the resulting microdosimetric spectra i.e. enhancing the high lineal energy component for relatively low energy ions. Aluminium was chosen as a top contact material whereas gold was chosen as a back contact to boron buried mesh.

Current design of microdosimeter is a solid state version of the 2D gas wire ionization chamber where boron implanted mesh is similar to wire anodes. This type of design will take advantage of high electric field that can be applied. The thin diamond layer $(1.38 \mu \mathrm{m})$ and high electric filed means that charge can be collected quickly before it can become trapped in defect centres typical for e-h transport in diamond.

\section{Methodology}

An important requirement of a microdosimeter is an accurately defined and well characterised charge collection volume. It is also important from a quality assurance point of view to assess the new microfabrication technique used to realise the $3 \mathrm{D}$ SVs.

In order to test these properties, an Ion Beam Induced Charge Collection (IBIC) study of the devices was undertaken on the Heavy Ion Microprobe (HIM) beam line on the ANTARES accelerator at the Australian Nuclear Science and Technology Organization (ANSTO). The purpose of this study was to ascertain the spatially resolved charge collection characteristics of the device. I-V characteristics of the device were measured to select bias for charge collection studies and effect of UV priming on them.

A Geant4 [11, 12] application was developed to characterise the diamond based microdosimeter in terms of effect of aluminium front contact thickness in proximity of the SVs, additionally to tissue equivalency in typical space radiation environment which is a subject of a separate paper.

\section{ELECTRICAL CHARACTERISTICS}

$\mathrm{I}-\mathrm{V}$ characteristics of the diamond microdosimeter were measured using conventional current-voltage (IV) testing with a Keithley 6517A electrometer at room temperature. Measurements were taken using a common back contact, pin 9 and front Al electrode connected to pin 20. This back contact being a gold contact that has been implanted using the FIB method mentioned previously. I-V measurements were taken in the range of $-15<\mathrm{V}<15$. A bias was applied to pin 9 and ramped in $0.5 \mathrm{~V}$ increments. Pin 20 was connected to a virtual ground corresponding to an input of electrometer. A 1s delay between each step was implemented in order to allow measured currents to stabilise.

I-V measurements were undertaken before and after exposure to UV light in order to examine whether there was an appreciable effect due to "priming". After the initial testing of the device, UV light was made incident for three hours upon the device and the electrical characteristics were retested. I-V measurements were taken after an hour and also after a week. Fig. 5 shows the results from IV measurements. The results presented show I-V characteristics before, 1 hour and 1 week after UV priming.

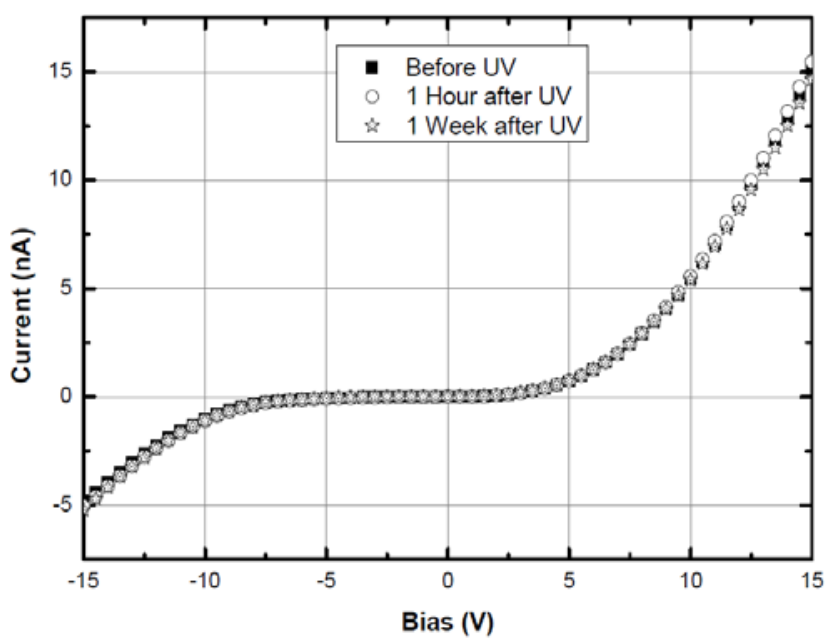

Fig. 5- IV Characteristics of the Diamond microdosimeter prototype as a function of time related to UV priming. All results are with connections made between pins 9 and 20

The results show that the effect of UV priming is negligible. This can be seen in the stability of the I-V response over time. The I-V characteristics are fairly symmetric about the zero, with some rectifying behaviour. The capacitance was found to be independent of the applied voltage as expected. Electrical characterization proved that the device behaves similarly to the solid state ionization chamber in contrast to SOI p-n junction based microdosimeter.

\section{IBIC EXPERIMENT}

The IBIC experiment was performed using a $5.9 \mathrm{MeV}$ mono-energetic beam of beryllium fully stripped ions, focused to a spot size of approximately $1 \mu \mathrm{m}$. The ion beam was normally incident on the microdosimeter device biased at $20 \mathrm{~V}$ 
and raster scanned across the device. The beam flux was maintained at $100 \pm 10$ ions per second throughout all experiments. The amount of energy deposited within the microdosimeter for each ion traversal, was measured with a standard charge sensitive preamplifier, shaping amplifier and multi-channel analyser (MCA) in coincidence with digitized voltage signals of the beam position, $\mathrm{x}$ and $\mathrm{y}$ for each event in $\Delta \mathrm{E}$. Data triplets $(\mathrm{x}, \mathrm{y}, \Delta \mathrm{E})$ were saved and stored. Analysis software was then used to generate IBIC imaging maps displaying a median energy map showing a spatially resolved image of the median amount of collected charge. The charge collection was investigated in different regions under the $\mathrm{Al}$ front electrode.

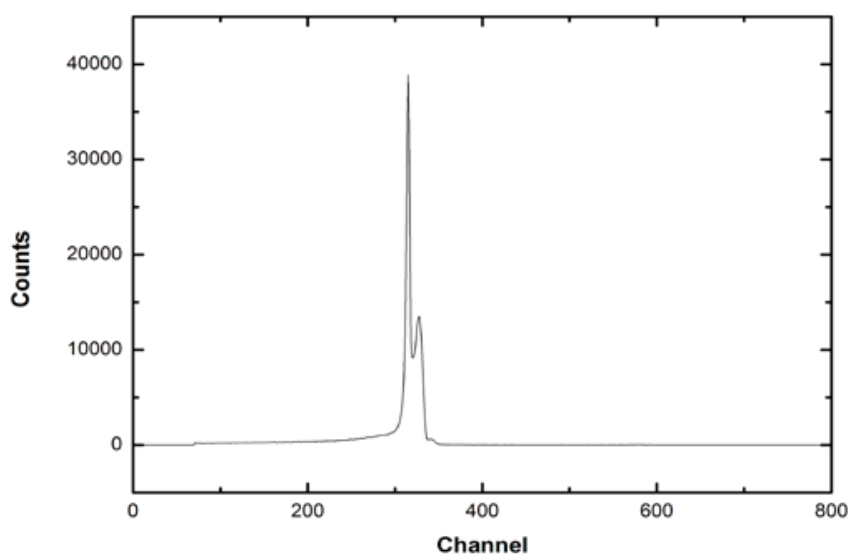

Fig. 6 a MCA spectrum generated by $5.9 \mathrm{MeV}$ beryllium ions directed onto a silicon detector. The two peaks are due to the energy loss in of the particles passing through the copper of the grid or unimpeded through the grid spaces.

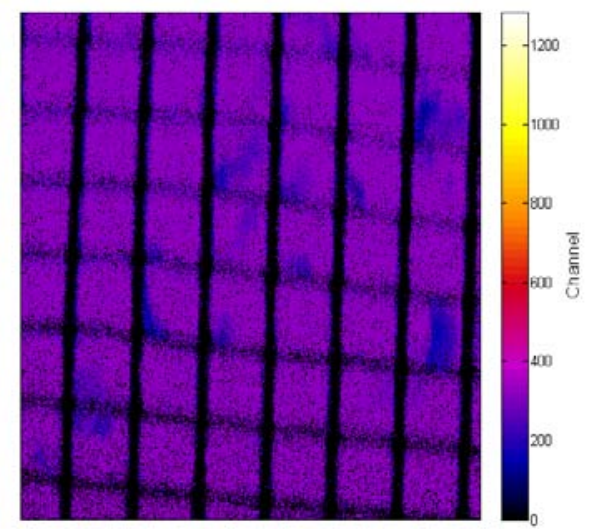

Fig.6b Scanning Transmission Ion Spectroscopy (STIM)
measurement of a $25.4 \mu \mathrm{m}$ pitch copper grid. This 1x scan amplifier measurement was used to spatially calibrate the IBIC images and determine the spot resolution. The image is $177.8 \mu \mathrm{m}$ in width.

\subsection{Calibration}

Fig. 6a shows the MCA spectrum collected by a B-series charged particle detector from ORTEC. This measurement is used for alignment of the beam as well as determining the scan area and beam spot resolution. The alignment consists in the scanning of a 1000 lines per inch copper grid placed in front of a silicon diode (Fig.6b) for verification of the positioning of the beam. The spectrum in Figure 6a shows a double peak due to the attenuation of the beryllium ions passing through the copper grid. Using the STIM measurement the size of the spatial charge collection features can be measured.

A Hamamatsu $300 \mu \mathrm{m}$ thick windowless silicon PIN diode has been used to calculate the calibration of the energy median maps of the diamond detector. The calibration factor has been calculated also taking into consideration the energy deposition of the primary ions (5.9 MeV) and the ratio of the energy required for production of a single e-h pair of silicon $(3.6 \mathrm{eV})$ and diamond $(13 \mathrm{eV})$. Final calibration factor for diamond taking into account the e-h production energy is $\mathrm{E}=0.4816$ $\mathrm{keV} /$ Channel with an offset of $-91 \mathrm{keV}$. This results in a calibrated energy of $390.6 \mathrm{keV}$ at Channel 1000. The maximum Charge Collection Efficiency (CCE) of the diamond detector is approximately $30 \%$. This is done by comparing the ratio of the maximum energy deposition approximately 565 $\mathrm{keV}$ to the theoretical energy deposition $1891 \mathrm{keV}$ of a 5.9 $\mathrm{MeV}$ Be ion in $1.38 \mu \mathrm{m}$ of diamond. Pixels underneath the middle of the aluminium pad have demonstrated a reasonable uniform charge collection, however enhancement of the collected charge in pixels is observed closer to the edge of $\mathrm{Al}$ electrode.

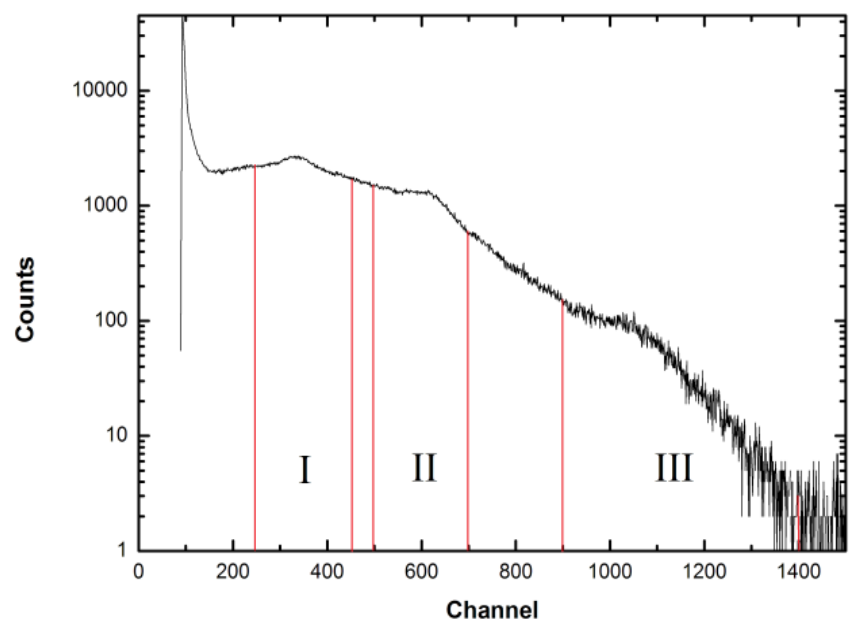

Fig. 7a MCA spectrum generated by $5.9 \mathrm{MeV}$ beryllium ions. The counts on the right hand side of the spectrum are those corresponding to the non-uniform charge collection in the IBIC map in Fig. 3.

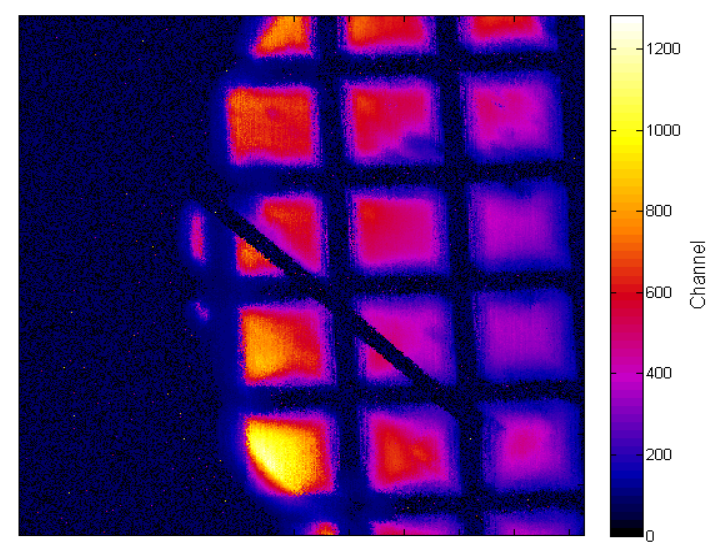

Fig.7b - Charge collection mapping of the device. These results come from charge collection towards the edge of the aluminium pad. Charge is collected only from beneath the aluminium pad. The image is $711 \mu \mathrm{m}$ in the $\mathrm{x}$ and $\mathrm{y}$ dimensions. 


\subsection{Charge collection study}

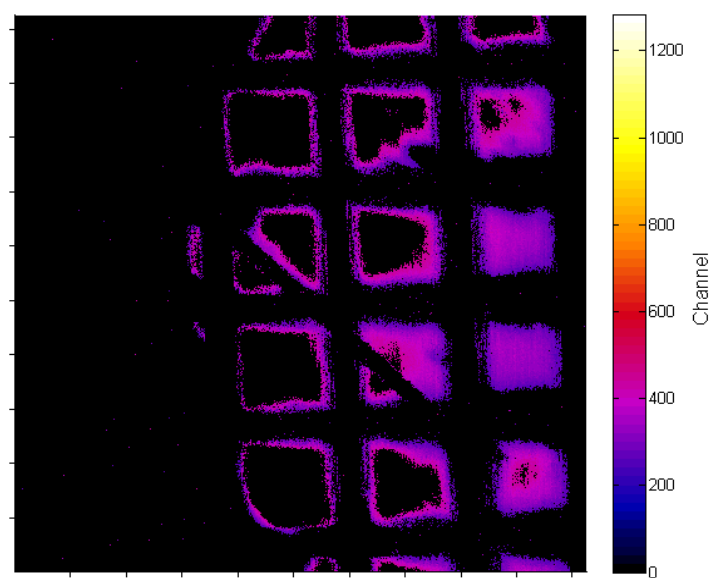

Fig.8 - Charge collection mapping of the device. These are the same results as from Fig. 7b, but the counts have been restricted energy window 30-126 keV. The most uniform charge collection is a third row.

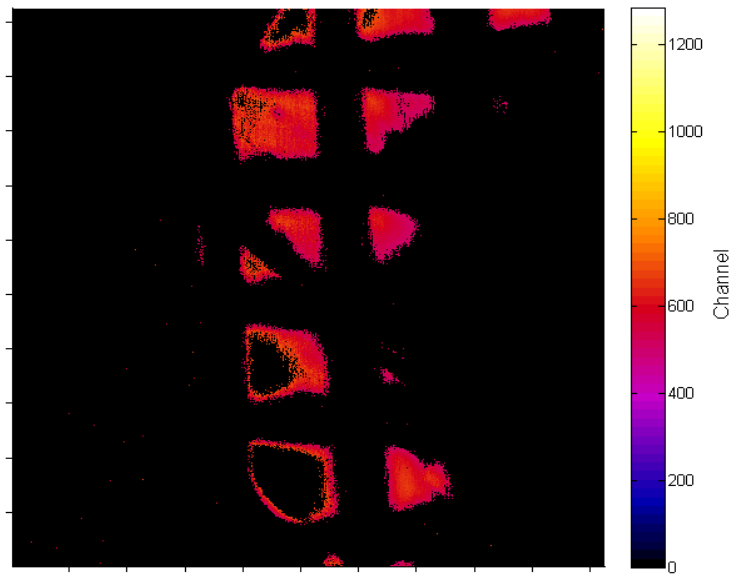

Fig.9 - Charge collection mapping of the device. These are the same results as from Fig. 7b, but the counts have been restricted for energy window 150-246 keV corresponding to the second 'peak' in Fig. 7a.

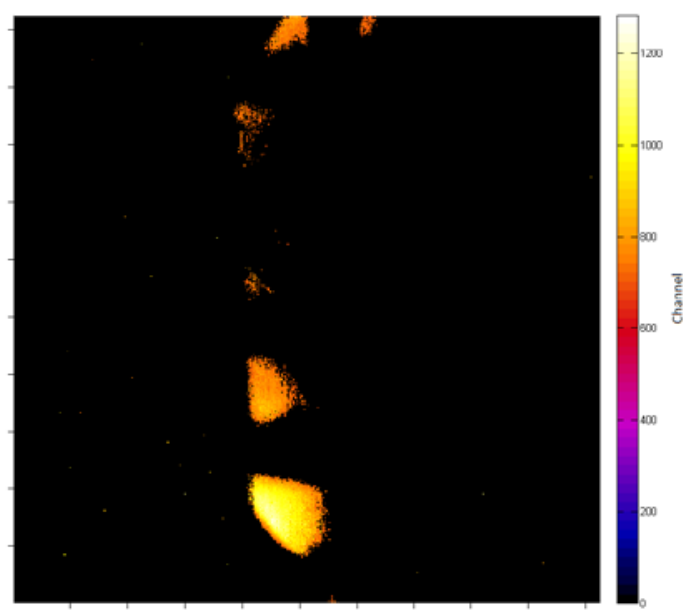

Fig.10 - Charge collection mapping of the device. These are the same results as from Fig. 7b, but the counts have been restricted to energy window 342-585 keV corresponding to the third 'peak' in Fig. 7a.

Detailed charge collection was investigated in a region close to the edge of $\mathrm{Al}$ front electrode. The MCA spectra and corresponding charge collection median energy map in terms of MCA channels are presented on a Fig 7a and Fig 7 b. respectively and obtained for scanning area about $0.7 \times 0.7$ $\mathrm{mm}^{2}$ as presented on a Fig $6 \mathrm{~b}$ during calibration with a $\mathrm{Cu}$ grid. The absence of charge collection between pixels is due to strong radiation damage from boron implantation as clearly observed.

The pixels close to the edge of $\mathrm{Al}$ electrode have demonstrated enhanced charge collection in comparison with those toward the centre corresponding to two peaks on MCA spectra. To better understand features on MCA spectra we have presented median maps corresponding to regions I, II and III on MCA spectra on Fig 8, 9 and 10 respectively.

Region I is concerned with channels in-between 250-450 and corresponds to an energy range of $\sim 30-126 \mathrm{keV}$ with a peak in the MCA spectra at channel 350 or rather $78 \mathrm{keV}$. The peak in the MCA spectrum would account for a CCE of approximately $4 \%$, taking into account the expect deposition of energy of a $5.9 \mathrm{MeV}$ beryllium ion in $1.38 \mu \mathrm{m}$ of diamond. However the spread of observed energy deposition suggests that charge collection within SV is not fully uniform and may be determined by a non uniform electric field distribution produced by $\mathrm{Al}$ electrode and buried implanted boron mesh. This uniform charge collection is more consistent toward the right in Fig 8, but less so in those SV's situated close to the edge of the Al front electrode. In those SV's that are situated close to the edge of the $\mathrm{Al}$ electrode, the charge collected specific to region I is located at the periphery of the pixels with the higher charge collection of regions II and III toward the centre.

The median map of charge collection corresponding to the region II on a MCA spectra which is corresponding energy range $150-246 \mathrm{keV}$ peaking around the channel $194 \mathrm{keV}$ is presented on a Fig 9 and demonstrating that these events are belong pixel located closer to the Al electrode edge. This second peak in region II gives a CCE of approximately $10 \%$. The difference in a charge collection can be explained partially with non uniform thickness of $\mathrm{Al}$ overlayer which is thicker and non uniform on an edge of the region. This is leading to an enhanced deposited energy in SVs due to the reduced energy of an ion and an increased electric field close to the edge of $\mathrm{Al}$ electrode.

Fig 10 shows a median map corresponding to region III on MCA spectra and related to small fragments in pixels in close proximity to the $\mathrm{Al}$ edge showing the highest amount of charge collection corresponding to an energy range of 342$585 \mathrm{keV}$. Due to the electric field distribution of the 3D SV configuration created by the boron mesh, there does exist at the periphery of these regions reduced charge collection specific to regions I and II. Lastly, the gold wire bonding is responsible for the lack of charge collection featured diagonally across the centre of Fig $7 \mathrm{~b}$. The gold wire bonding is of sufficient thickness $(\sim 25 \mu \mathrm{m})$ that beryllium ions are stopped.

The charge collection maps presented in Figs 7 through 10 show well defined 2D SV's limited by the boron buried grid.

To investigate the effect of a non-uniform aluminium overlayer, the diamond microdosimeter structure has been studied by Geant4 simulations. 


\section{THE GEANT4 SIMULATION}

The diamond microdosimeter was modelled in detail in the Geant4 9.3 application. A sketch of the device modelled in the experimental set-up of the simulation is shown in Fig. 11 and 12. The device is set within a vacuum. The device has been modelled to have four sensitive volumes of pure diamond 150x150x1.38 $\mathrm{\mu m}^{3}$; these are surrounded by a boron doped layer $480 \times 480 \times 1.38 \mu^{3}$. Underneath is a pure diamond layer of $480 \times 480 \times 300 \mu^{3}$. Above the sensitive volumes is aluminium pad $480 \times 480 \times T_{d} \mu m^{3}$, where $T_{d}$ is a variable thickness from 0 to $1 \mu \mathrm{m}$.

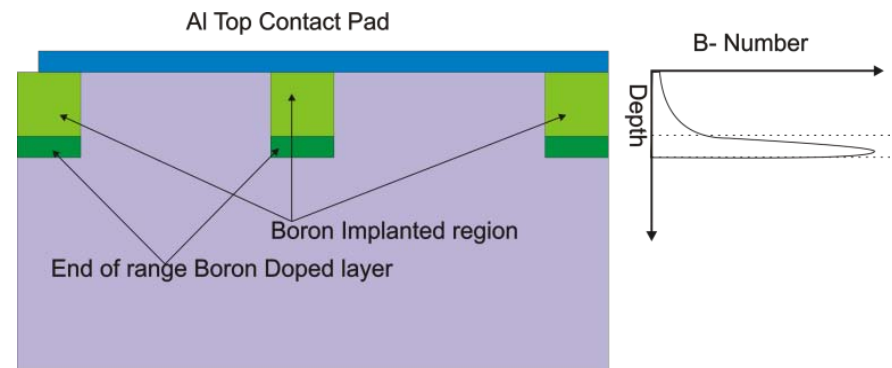

Fig. 11- Cross section of the Geant4 model of the diamond microdosimeter.

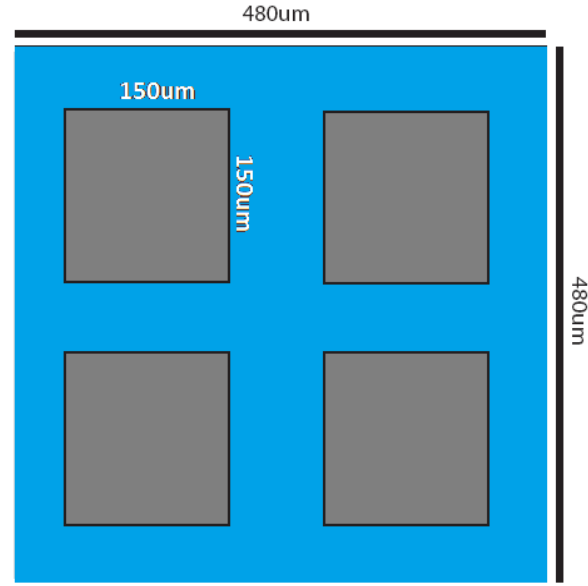

Fig. 12 - Top view of the Geant4 model of the diamond microdosimeter. The four diamond SV's lay beneath the $\mathrm{Al}$ layer but for clarity are made visible here.

The Geant4 General Particle Source (GPS) is used to model the different radiation fields adopted in the simulation study.

Two different radiation fields were modelled during the course of this study. The first was a pencil beam normally incident upon the centre of a SV.

The Geant4 Low Energy Physics Package, based on Livermore data libraries [13] was employed to describe all the electromagnetic interactions of particles, valid down to 250 $\mathrm{eV}$. The energy threshold of production of secondary particles was fixed equal to a 10 nanometres cut in range corresponding to $250 \mathrm{eV}$, to describe the interactions of particles with adequate accuracy.

The Geant4 QGSP_BIC_HP physics list was selected to model the hadronic physics processes because it has shown the best agreement with experimental results, in a proton radiation field of interest for our work [14].

Primary and secondary particles were tracked in the experimental set-up and the output of the simulation was the energy deposition per event in the SVs, where one event corresponds to the generation of one primary particle.
The Geant4 application was used to verify if the non-uniform charge collection, documented in section IV, was due to a varying thickness of the aluminium pads. A pencil beam of stripped beryllium ions, with same energy of IBIC experiment, was normally incident on the microdosimeter device. The energy deposition per event was simulated for different thicknesses of the aluminium pad, ranging between 0 and 1 $\mu \mathrm{m}$. Fig. 13 shows the frequency of energy depositions deriving from $10^{5}$ events within the SV's with $0 \mu \mathrm{m}, 0.5 \mu \mathrm{m}$ and $1 \mu \mathrm{m}$ thick $\mathrm{Al}$ contact, respectively. The area of the plots is normalised to 1 . The peaks for $0 \mu \mathrm{m}, 0.5 \mu \mathrm{m}$ and $1 \mu \mathrm{m}$ thick Al contact are approximately $1.9,1.95$, and $2 \mathrm{MeV}$ respectively.

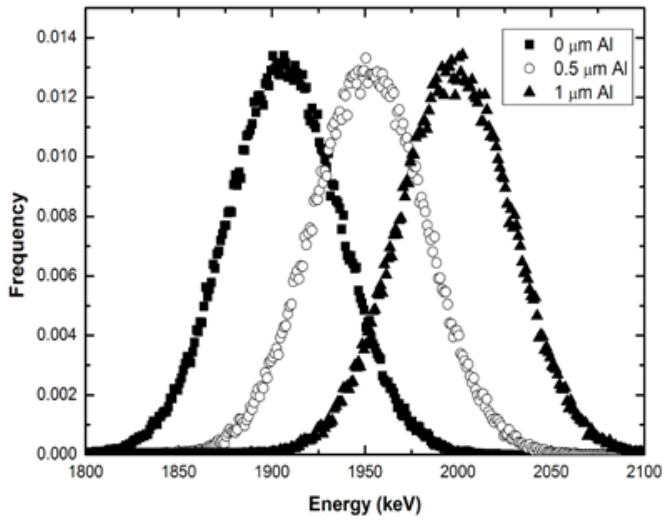

Fig. 13 - Energy deposition per event deriving from 5.9 MeV Be ions. The area of the curves is normalized to 1. Errors are shown but are small and fall within the symbol shapes.

Due to increased LET there is a shift in the energy deposition peak as the thickness of the aluminium pad is increased about $5 \%$ per 1 um of deposited $\mathrm{Al}$ for $5.9 \mathrm{MeV}$ Be ions. The effect of non uniformity of charge collection observed in SVs close to the edge of $\mathrm{Al}$ electrode demand much higher than $1 \mu \mathrm{m}$ variation of $\mathrm{Al}$ thickness to explain charge enhancement almost twice for regions presented in a Fig. 10 in comparison with Fig 9. Further understanding of this edge effect should be carried out.

\section{DISCUSSION AND CONCLUSION}

The Ion Beam Induced Current experiments showed that the novel technique for the production of a buried boron conducting mesh, using $2 \mathrm{MeV}$ boron implantation through an $\mathrm{Al}$ mask in a detector grade CVD diamond substrate leads to well defined $3 \mathrm{D}$ vertical SVs. The advantage of this technology is in the relative simplicity of design. The utilisation of this technology removes the necessity for any additional overlayers such as that used in p-n junction array formation as in SOI microdosimeter. Another advantage of a diamond microdosimeter produced through high energy ion impanation is in a single Al electrode on the top of the device, which enables a simple connection of the array of sensitive volumes. The electrode improves the reliability of the device in comparison with SOI devices based on planar p-n junctions where the connection of the sensitive volumes demand thin $\mathrm{Al}$ leads produced by photolithography.

Underneath the majority of the Al electrode, uniform charge collection was achieved. However full charge collection corresponding to ideal $1.38 \mu \mathrm{m}$ thickness of diamond 3D SV 
was not observed. A gradual increase in charge collection efficiency is observed towards the edges of aluminium contact pad. Given the range of a $5.9 \mathrm{MeV}$ beryllium ion in diamond and aluminium of 4.36 and $8.26 \mu \mathrm{m}$ respectively, it has been hypothesised that the non uniformity in charge collection may be partially explained by a variance in the thickness of the overlaying aluminium layer [15]. A study using both Geant4 and SRIM based simulations has shown that while small variations in the $\mathrm{Al}$ overlayer can have a small effect upon the energy deposition within the sensitive volume, it is not enough to account for the change in charge collection efficiency seen in the IBIC results. This has led to the conclusion that the increased charge collection efficiency of region III in comparison with region I (in Fig 7a) may result from material and electric field non uniformity in the 3D SVs under the Al electrode as opposed to dimensional variations in the $\mathrm{Al}$ electrode itself.

The optimization of the proposed technology in the forming of the array of 3D sensitive volumes in CVD diamond can be achieved by through a modelling of the electric field structure of the device using ISE-TCAD and will be the subject of future studies. This concept will also be studied experimentally using IBIC with a range of ions of different initial energies and LET to investigate any funnelling effects downstream of buried boron mesh and the true depth of the sensitive volumes produced by the built in electric field. Room temperature annealing can lead to changing of the geometry of the sensitive volume and also should be investigated. In future work, it shall be investigated whether or not the near four times less charge induced for the same deposited energy in comparison with silicon will significantly compromise the dynamic range of the detected lineal energies of low LET ions. The next step will be to realise true microdosimetric volumes using this established fabrication technique of creating isolated wall-less sensitive volumes using boron ion implantation radiation damage.

In conclusion a novel diamond microdosimeter design has been described with a prototype device modelled using the Geant4 toolkit and characterized in terms of its charge collection properties and isolation of the micron-sized sensitive volumes. Such a design potentially offers advantages over the well-accepted SOI microdosimetry technology, including radiation hardness and soft tissue equivalence. The results presented indicate the viability of the design that utilizes radiation damage through boron ion implantation, as a means of creating isolated wall-less sensitive volumes within the diamond structure. This fabrication technology therefore looks very promising for use in future diamond based microdosimeter devices to be developed at CMRP in collaboration with UOM.

\section{ACKNOWLEDGEMENTS}

This research was supported by Australian Research Council Discovery Grant ID 1096600 and partially by grant from National Space Biomedical Research Institute (NSBRI), USA.

\section{REFERENCES}

[1] H. H. Rossi and M. Zaider, Microdosimetry and Its Applications. London: Springer, 1996
2] P.D.Bradley, A.B.Rosenfeld, M.Zaider (invited paper), "Solid State Microdosimetry", Nucl.Instr.Meth. in Phys.

Research B, 184, 135-157, 2001

[3] W. Adam, et al, Radiation hard diamond sensors for future tracking applications, Nucl. Instrum. Meth. A, Vol. 565(1), pp. 278-283, 2006.

[4] L.S. Pan, D.R. Kania (editors), "Diamond: Electronic Properties and Applications”, Kluwer Academic Publishers, Boston/Dordrecht/London, 1995

[5] Frais-Kolbl, H.; Griesmayer, E.; Kagan, H.; Pernegger, H.; , ”A fast low-noise charged-particle CVD diamond detector," IEEE Trans. Nucl. Sci. , Vol.51(6), pp. 3833- 3837, 2004.

[6] S.V. Akulinichev, V.S. Klenov, L.V. Kravchuk, S.G. Lebedev, A.V. Feschenko, V.E. Yants "Development of the charge particle detector based on CVD-Diamond" Institute for Nuclear Research of the Russian Academy of Sciences, 60-th October Anniversary Prospect, 7A, Moscow, 117312

[7] C Manfredotti, P Polesello, M Truccato, E Vittone, A Lo Giudice, F Fizzotti, CVD diamond detectors, Nucl. Instrum. Meth. A, Volume 410(1), pp. 96-99, 1998.

[8] A, Brambilla et al, Thin CVD Diamond Detectors With High Charge Collection Efficiency, IEEE Trans. Nucl. Sci. Vol. 49(1), 2002

[9] M. Mathes et al, Characterization of a single crystal diamond pixel detector in a high energy particle beam, IOP PUBLISHING AND SISSA 2008.

[10] Element Six. (2012, January, 10). CVD synthetic diamond [Online]. Available: http://www.e6.com/

[11] S. Agostinelli et al., Geant4A simulation toolkit, NIM A, Vol. 506(3), 2002, pp. 250303.

[12] J. Allison et al., Geant4 developments and applications, IEEE Trans. Nucl. Sci., Vol. 53(1), 2006, pp. 270278.

[13] S. Chauvie et al., Geant4 low energy electromagnetic physics, IEEE Nuclear Science Symp. Conf. Rec., vol. 3, pp. 18811885, 2004.

[14] C. Zacharatou Jarlskog and H. Paganetti, Physics settings for using the Geant4 toolkit in proton therapy, IEEE Trans. Nucl. Sci., Vol. 55, 2008, pp. 1018-1025.

[15] Berger, M.J., Coursey, J.S., Zucker, M.A., and Chang, J. (2005), ESTAR, PSTAR, and ASTAR: Computer Programs for Calculating Stopping- Power and Range Tables for Electrons, Protons, and Helium Ions (version 1.2.3). [Online] Available: http://physics.nist.gov/Star [2011, April 10]. National Institute of Standards and Technology, Gaithersburg, MD. 